\title{
The microwave subsecond pulse of September 17, 2001: The spectrum, location and size of the source
}

\author{
A. T. Altyntsev ${ }^{1}$, S. V. Lesovoi ${ }^{1}$, N. S. Meshalkina ${ }^{1}$, R. A. Sych ${ }^{1}$, and Yihua Yan ${ }^{2}$ \\ 1 Institute of Solar-Terrestrial Physics, Siberian Branch of the Russian Academy of Sciences (ISTP), PO Box 4026, \\ Irkutsk 33, 664033, Russia \\ e-mail: altyntsev@iszf.irk.ru \\ 2 National Astronomical Observatories, Chinese Academy of Sciences, Datun Road A20, Chaoyang District, \\ Beijing 100012, China \\ e-mail: yyh@bao.ac.cn
}

Received 8 July 2002 / Accepted 13 December 2002

\begin{abstract}
We examine simultaneous observations of microwave subsecond pulses with high temporal, spatial and spectral resolution from the Siberian Solar Radio Telescope (5.7 GHz, $14 \mathrm{~ms}$ resolution) and from the spectropolarimeters $(5.2-7.6 \mathrm{GHz}$, $6 \mathrm{~ms}$ ) of the National Astronomical Observatories. The September 17, 2001 flare is discussed in detail. The subsecond pulse (SSP) was observed in the initial phase of the flare, and its HWFH duration was $40 \mathrm{~ms}$. The pulse was accompanied by a rapid change of the dynamic spectrum whose width was about $1 \%$, and the degree of polarization made up $35 \%$. The time profile at the SSRT frequency depends substantially on the SSP's spectral features. The SSP was observed by both the NS (NorthSouth) and EW (East-West) arms (in two interference orders in the latter case). SSP sources locations in burst structures were determined. We also find that the source was not a point-like one, but its apparent size was about the beam width (15 arcsec) for the NS scanning direction. In the EW direction the SSP was less than 10 arcsec in size.
\end{abstract}

Key words. Sun: flares - Sun: radio radiation

\section{Introduction}

It is generally accepted that the study of structures of a subsecond duration in radio emission holds promise for investigating the processes of primary energy release of solar flares. Of special interest are narrow-band bursts, or spikes, associated with nonthermal coherent mechanisms of emission. Their effectiveness and the very possibility of their realization depend strongly on plasma parameters: the magnetic field, density, and accelerated particle distributions (Benz 1986; Fleishman \& Melnikov 1998). If the emission mechanism is known, then spike observations make it possible to estimate the plasma parameters in the source which are inaccessible by other methods. A central problem encountered when determining the emission mechanism is the small number of observations combining temporal, spatial and spectral resolutions.

The expected source size of narrow-band spikes of the microwave range does not exceed several hundred kilometers. First observations with the two-element interferometer at $2.8 \mathrm{GHz}$ provided the upper estimate of the size of $26^{\prime \prime}$

Send offprint requests to: $\mathrm{N}$. S.Meshalkina, e-mail: nata@iszf.irk.ru
(Gary et al. 1991). They were followed by VLBI observations of 26 events with fine temporal structure at $2.3 \mathrm{GHz}$ which gave the lower estimate of the source size of $65 \mathrm{~km}$ (Benz et al. 1996). The frequency of $5.7 \mathrm{GHz}$ was used to carry out a number of observations of the source size of subsecond impulses with the Siberian Solar Radio Telescope (SSRT) in the one-dimensional mode with a temporal resolution of $56 \mathrm{~ms}$ (Altyntsev et al. 1996a). It was found that the apparent source size is $10-50^{\prime \prime}$ and depends on source position on the solar disk, reaching maximum values for limb events. The increase of the size toward the limb was explained by the scattering of emission on plasma inhomogeneities in the Sun's lower corona. $17 \mathrm{GHz}$ observations with subsecond temporal resolution are feasible with the Nobeyama radioheliograph. Estimates of the source size of subsecond pulses made by Shibasaki et al. (1994), Takano et al. (1994), Altyntsev et al. (2000) did not exceed the width of the NoRH beam (about $10^{\prime \prime}$ ).

Spatially resolved observations are crucial for determining the generation mechanism of the fine temporal structure. They make it possible to localize the source in the flaring region and, using data for other emissions, to estimate the plasma parameters in the region of its generation. 


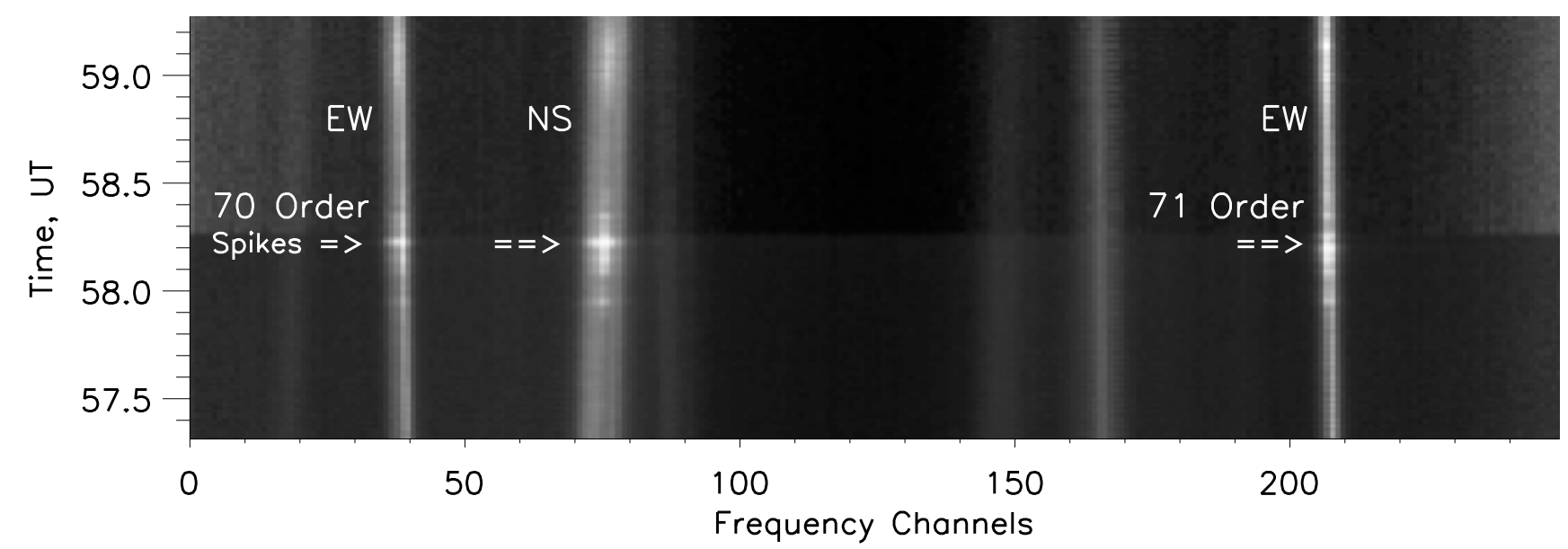

Fig. 1. Sequence of the one-dimensional records for the flare on September 17, 2001. Time range 08:20:57.3-08:20:59.3. The SSRT beam widths were $18.2^{\prime \prime}$ and $24.3^{\prime \prime}$ for NS and EW, respectively. The angular spaces between neighboring beams were $10.5^{\prime \prime}$ and $19.6^{\prime \prime}$. The strip inclinations was caused to the relative Earth-Sun movement.

Until now interferometric observations have been carried out at a single working frequency and without simultaneous observations of the dynamic spectrum of the microwave burst. Regular observations with the spectropolarimeters of the Beijing observatory ( $\mathrm{Fu}$ et al. 1995) provided a means of carrying out simultaneous observations with spatial resolution (SSRT), and with spectral resolution (NAOC). The peculiar feature of the September 17, 2001 event was the combination of the data on the dynamic spectrum with interferometric observations in two directions. Moreover, the source was observed in two frequency ranges.

An added interest in this event is the high degree of circular polarization of subsecond pulses. Before 2000, the SSRT temporal resolution $(56 \mathrm{~ms})$ was inadequate for polarization measurement of pulses when their duration was shorter than $0.5 \mathrm{~s}$ (Altyntsev et al. 1996b). From June 2, 2001 to April 14, 2002, 54 events were recorded with subsecond pulses with a resolution of $14 \mathrm{~ms}$, and 20 events of them showed the polarization degree over $30 \%$.

\section{Methods of observation}

The SSRT consists of two perpendicular lines of antennas: east-west (EW), and north-south (NS), operating in the frequency range 5.67-5.79 GHz. Radio maps of the solar disk are recorded at intervals of 3-5 min. The fine temporal structure of flare bursts is analyzed using data recorded by the EW and NS arrays separately which provide one-dimensional scans of the solar disk every $14 \mathrm{~ms}$ (Fig. 1). The components of the circular polarization ( $\mathrm{R}$ and $\mathrm{L}$ ) are recorded successively within the interval of $7 \mathrm{~ms}$ duration each.

The technique for analyzing one-dimensional solar images was described by Altyntsev et al. (1996a). The SSRT receiving system comprises the spectrum analyzer in the $120 \mathrm{MHz}$ frequency band which is represented by the acousto-optical receiver. Its 250 frequency channels correspond to the fan of knife-edge beams for the NS and EW arrays, respectively. The band width of one frequency channel is $0.48 \mathrm{MHz}$. The response at each frequency corresponds to the emission from a narrow strip on the solar disk whose position and width depend on the time, and the type of array. Signals from all channels provide an instant one-dimensional distribution of radio brightness. Spatial resolution depends both on the beam width and on the angular space between neighboring knife-edge beams.

In the event under consideration, the angles between knifeedge beams and the central meridian were 24.41 degrees (EW) and -21.88 (NS), and the beam widths were $18.2^{\prime \prime}$ and $24.3^{\prime \prime}$ for NS and EW, respectively. The angular spaces between adjucent beams were $10.5^{\prime \prime}$ and $19.6^{\prime \prime}$ for NS and EW, respectively. Given the small size of microwave bursts, they are recorded by the SSRT in a narrow band not exceeding $5 \mathrm{MHz}$.

In some intervals of local time the SSRT observes the flare region in two interference maximums (orders) simultaneously. Interference maximums with numbers $n=0, \pm 1, \pm 2, \ldots$ occur in the directions $\vartheta$, where $\cos \vartheta=\frac{n c}{v d}$, with $c$ being the speed of light and $d$ the fundamental spacing of the interferometer. Different interference maximums are observed in the same directions $\vartheta_{0}$ at different frequencies if $\frac{n_{i}}{v_{i}}=\frac{n_{0}}{v_{0}}=d \cos \vartheta / c$. Two solar images formed in two adjacent interference numbers are observed simultaneously in different frequency channels when the interference numbers, $n_{0}>v_{0} / \Delta v$, where $\Delta v$ is the operating band width of the SSRT. The September 17 event was observed in 70th and 71st orders simultaneously.

Dynamic spectra were observed with Solar Radio Broadband Fast Dynamic Spectrometers (5.2-7.6 frequency range) at Huairou Solar Observing Station of the Beijing Astronomical Observatory (Fu et al. 1995). The reception band of an individual frequency channel of the NAOC spectropolarimeter is $20 \mathrm{MHz}$, and the temporal resolution is $5.9 \mathrm{~ms}$.

\section{Observations}

Figure 2 shows the data on a subsecond pulse. The frequency of observation of the SSP source by the NS array differed from the EW frequency in the 70th order by $17.26 \mathrm{MHz}$, and the difference between the channels in adjacent orders of EW was 80.16 MHz. 

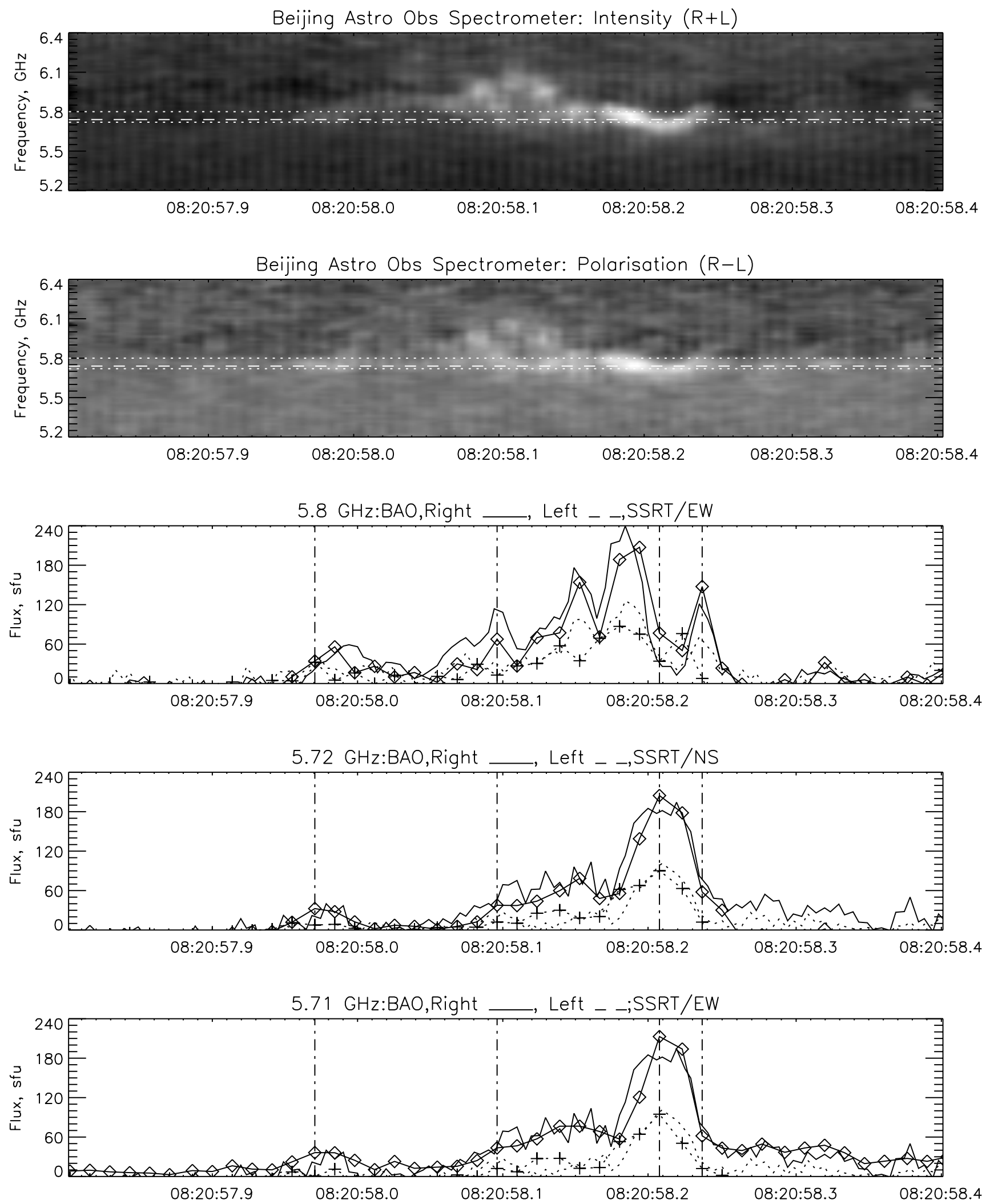

Fig. 2. Spectral and temporal characteristics of the subsecond pulse. The upper panels show the dynamic spectrum from NAOC in the intensity $(\mathrm{R}+\mathrm{L})$ and polarization $(\mathrm{R}-\mathrm{L})$ in the frequency range 5.2-6.4 GHz. Horizontal lines correspond to the frequency channels which recorded SSP at the SSRT (EW, NS, and EW, from top to bottom). Below, the profiles of the fluxes recorded with the spectropolarimeter in the R and L circular polarization at the SSRT reception frequencies, and lines with diamonds and crosses correspond to the SSRT fluxes obtained by integrating one-dimensional distributions over the SSP source. The diamonds and crosses show the time of recording one-dimensional distributions with the SSRT. 

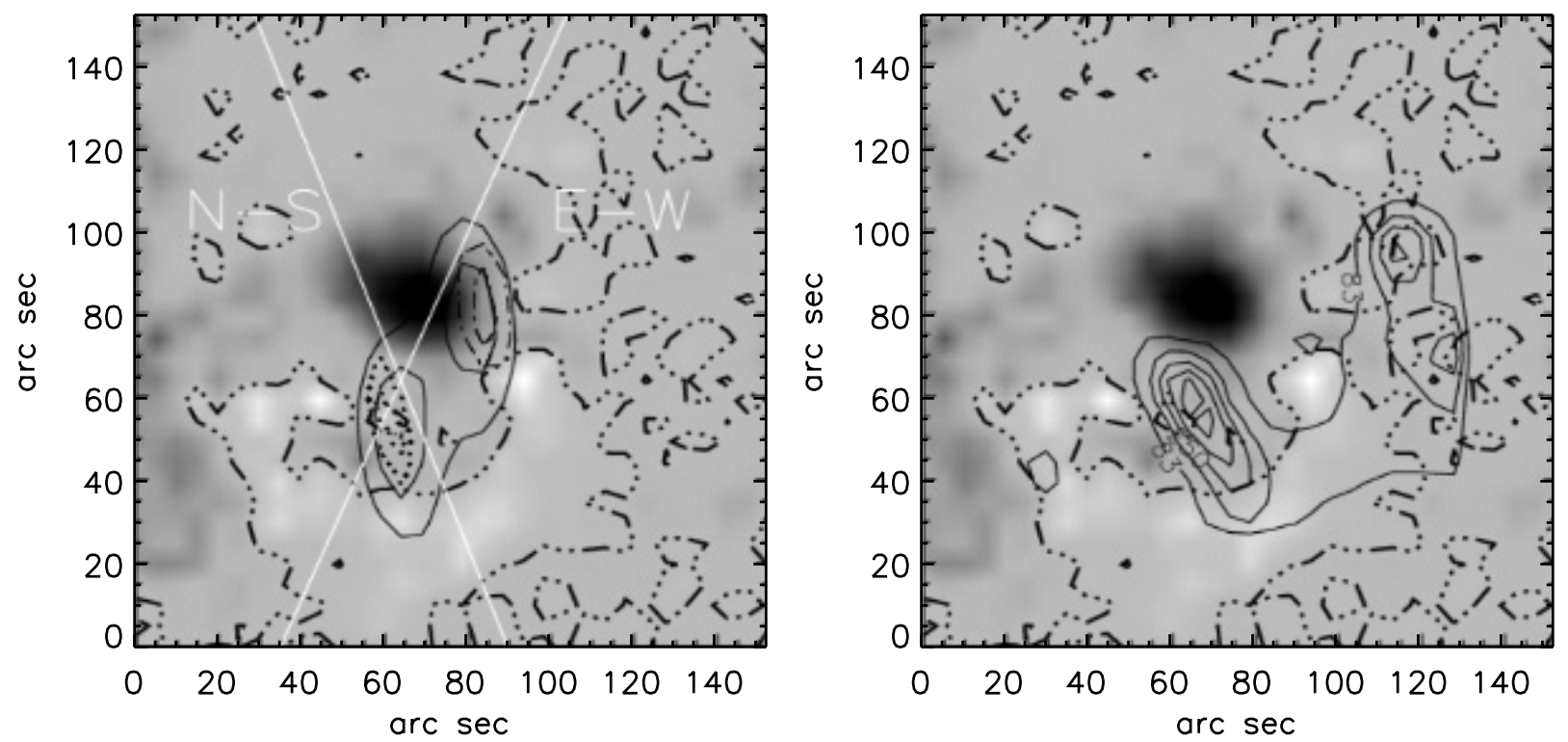

Fig. 3. Structure of the flaring region. The background is represented by the MDI magnetogram taken at 08:03:30 UT. The dash-dotted line shows the neutral line of the photospheric magnetic field. Left: structure of the microwave burst at 08:20:38 UT. Contours show the brightness temperatures in the intensity $(\mathrm{R}+\mathrm{L})$ by solid lines, and in the polarization $(\mathrm{R}-\mathrm{L})$ : (dotted - RCP, dash-dotted - LCP). White lines are along one-dimensional beams and perpendecular to scanning directions. The intersection of the lines corresponds to the SSP brightness center. Right: contours show the brightness distribution of SXR emission as measured by Yohkoh/SXT (AlMg) at 08:21:06 UT. North - upward, West - to the right.

The dynamic spectrum shows the pulse duration of $0.25 \mathrm{~s}$, with the mean frequency around $6 \mathrm{GHz}$. The U-shaped form of the spectrum dynamics corresponds to the interval with the brightest emmision. The total frequency range reaches $5 \%$, and near the peak the spectral width is less than $50 \mathrm{MHz}$, or $1 \%$ of the receiving frequency. The value of the integral flux reaches 220 sfu at maximum SSP, and the degree of circular polarization of integral emission is as large as $35 \%$.

It is evident that the time profile of the signal recorded by the SSRT is determined by the dynamics of the spectrum in frequency. The agreement between the NAOC and SSRT signals is quite satisfactory, considering the difference in the band width of received signals, and the noise influence. We see a good agreement between SSRT signals from the NS and EW arrays, the receiving frequencies of which are close.

$\mathrm{X}$-ray emission of the flare began to increase at 8:20:20 UT (Yohkoh/HXT/L). The flare of class M1.5 occurred in the central part of the solar disk (S12E06) in the active region of a $\beta \gamma$-configuration (NOAA 9616). The subsecond burst was observed at the beginning of the flash stage of the flare during a slight decrease in brightness. From the value of the ratio of signals from the Yohkoh/HXT channels follows the estimate of the spectral index of about 3-4. Unfortunately, the Yohkoh/HXT time resolution of $0.5 \mathrm{~s}$ is insufficient for studying the correlations of the fine structure of microwave and hard X-ray emissions.

Two-dimensional images from the SSRT were available at 08:18:50, 08:20:38 and 08:22:12 UT. It is evident from Fig. 3 that at 08:20:38 UT the microwave sources represent a bipolar structure extended in a meridional direction: the northern LCP source lies above the sunspot of S-polarity, and the southern $\mathrm{RCP}$ source is located above the sunspot of N-polarity.
The magnitudes of the magnetic fields in the sunspots reach $-1750 \mathrm{G}$ and $370 \mathrm{G}$, and the degrees of polarization are as large as $-35 \%$ and $12.5 \%$, respectively. The distance between the polarization centers is $30-35$ arcsec.

The previous image, prior to the flare, showed one LCP microwave source between magnetic field spots. Consequently, the sources in Fig. 3 have their origins in the flare and appear with an increase in X-ray emission of accelerated electrons.

The sources of the background burst are seen separately in NS one-dimensional images and blend on the EW distributions. Figure 3 shows also the directions of the knife-edges of the one-dimensional beams at the time of recording the SSP. Onedimensional brightness distributions were not available before 08:20:51 UT. At this time the both flare sources were seen in intensity (R+L), but the southern RCP source (Fig. 3) has reversed the sign of polarization.

Contours in the right-hand panel show the soft X-ray map which is available $8 \mathrm{~s}$ after the SSP. In the horseshoe-shaped structure encompassing the flaring region from the south, the region near the southern source is the brightest. An estimation of the plasma parameters during the SSP using GOES data gives the temperature of $1.2 \times 10^{7} \mathrm{~K}$, and the emission measure of $6.2 \times 10^{48} \mathrm{~cm}^{-3}$. With the source size $10^{\prime \prime} \times 10^{\prime \prime} \times 20^{\prime \prime}$, the plasma density is estimated at $2 \times 10^{11} \mathrm{~cm}^{-3}$.

A sequence of one-dimenshional NS brightness distributions (scans) for the instants of time shown by vertical lines in Fig. 2, is shown in Fig. 4. The increments in L emission are significantly smaller than those for the $\mathrm{R}$ component.

The spatial resolution is insufficient for determing the brightness center using scan maximums. Centers of gravity of one-dimenshional brightness distributions are therefore used. Figure 5 shows the positions of the centers. The first interval 


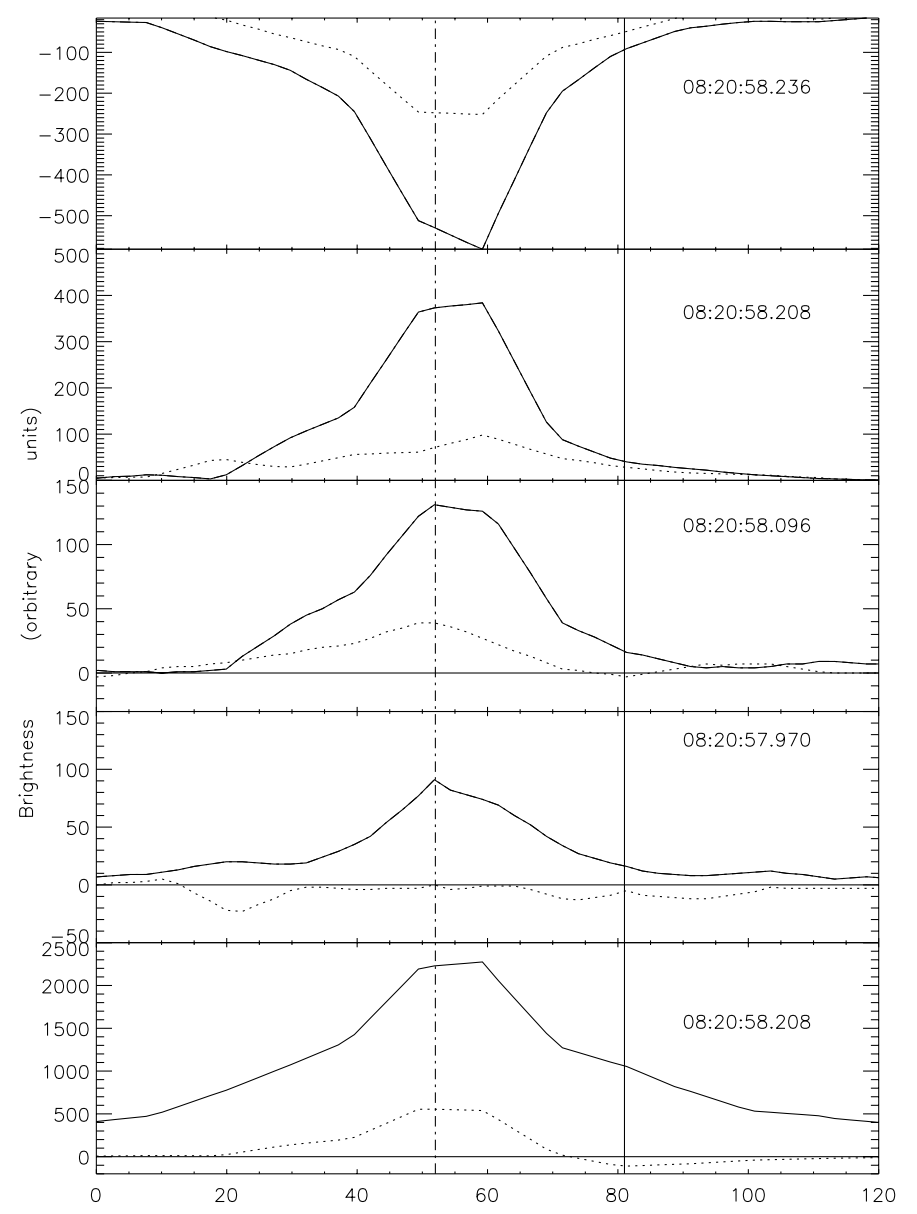

Fig. 4. Sequence of NS scans. The lower panel presents the distributions of the R+L emission intensity (solid line) and the R-L polarization (dotted line) at the SSP maximum. The upper panels present the difference scans of R (solid line), and L (dotted line) obtained by subtracting from the scans, shown by the vertical lines (Fig. 2), the preceding ones (14 ms before). Vertical lines show the positions of the brightness centers of the background burst: solid line for the LCP component, dash-dotted line for the RCP component.

corresponds to the position of the background burst, and the second one refers to the SSP source. It is evident from the second panel corresponding to the NS array that the position of the source of right-handedly polarized emission is constant during the SSP, and is displaced northward from the background burst by $6^{\prime \prime}$. The displacement of the left-handedly polarized source is significantly less.

Scans in Fig. 4 are the result of a convolution of the microwave emission source with the NS beam. By approximating the form of the source and beam by the Gaussian distribution, the source size at the half-amplitude can be estimated at $\sqrt{\Delta^{2}-d^{2}}$, where $\Delta$ is the width at the half-height of the recorded response, and $d$ is the beam width of the interferometer. For the scans in Fig. 4, this estimation gives the source size of about $20^{\prime \prime}$. The analysis of the spatial structure of the SSP sources is detailed in Fig. 6.

An identical apparent size of SSP source can be obtained for two point-like sources with the distance between the sources of $15^{\prime \prime}$ along the NS scanning direction.

It is evident that the main SSP signal power is received in two frequency channels, the signal ratio of which is determined by the position of the center of gravity of the SSP source with respect to the receiving bands of the channels. Thus the SSP source is received by the NS array within a width less than $2 \mathrm{MHz}$ (less than $4 \times 10^{-4}$ of the receiving frequency), and the influence of the spectral features on the source size can be neglected.

The EW scans at $5.71 \mathrm{GHz}$ close to the NS receiving frequency, are shown in Fig. 7. In this case the angular distance between sections of the solar disk recorded by neighboring channels was about $20^{\prime \prime}$. The source size in both orders is significantly smaller than the EW beam and does not exceed $10^{\prime \prime}$.

The closeness of the vertical lines to one another shows that the background burst sources lay along the EW scanning direction.The brightness center of the SSP was close to the southern source of the background burst at the minimum frequency of the U-shaped structure. The behavior of the increments of the $\mathrm{R}$ and $\mathrm{L}$ distributions is similar to the corresponding scans in Fig. 4 for the NS array.

This case corresponds to the third panel in Fig. 5. A maximum displacement of the SSP source of about $2^{\prime \prime}$ (to the south) is observed for the R-component at the time of the U-structure minimum. The first panel shows similar dependencies for the L-component for EW sections, but higher in the receiving 


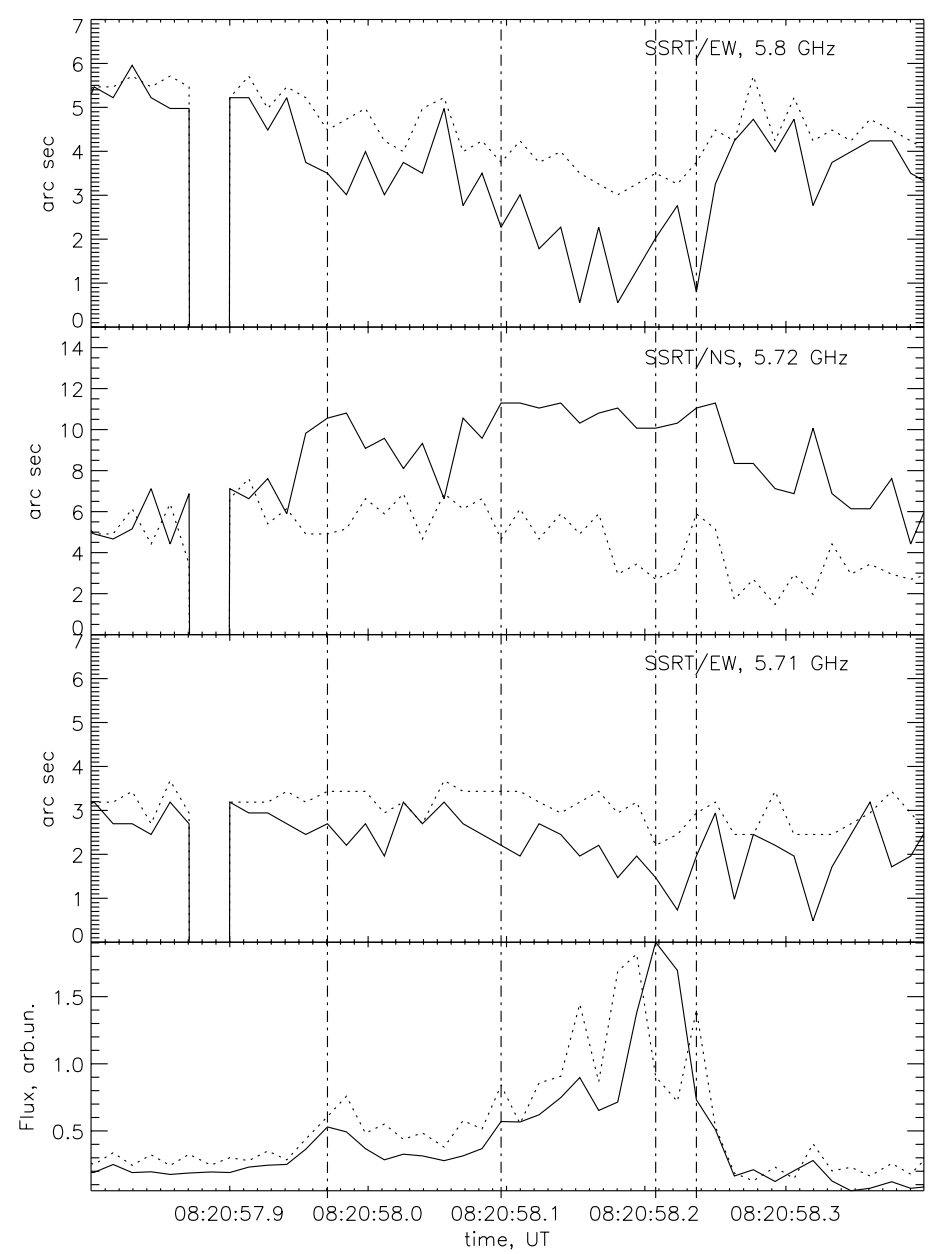

Fig. 5. Time profiles of the displacements of the centers of gravity of one-dimensional brightness distributions. Before 08:20:57.9 - shown are the centers of the background burst. After the gap in the data, shown are the centers of the SSP source calculated from the instantaneous scans and extracted scan of the background burst. The solid and dashed curves correspond, respectively, to the right- and left-handedly polarized emission. The lower panel shows the time profiles of Fig. 2: solid line for the NS array, dashed line for EW (71th order).

frequency by $80 \mathrm{MHz}$ (71st order). In this case there is a gradual displacement of the source. At the time of the U-shaped structure it reaches $4^{\prime \prime}$.

\section{Discussion}

\section{Comparison of spectrographic and interferometric data}

The pulse duration is $250 \mathrm{~ms}$, and the dynamic spectrum exhibits faster structures. The amplitude increases at the end of the pulse when the U-shaped structure is seen in the dynamic spectrum. The spectral width totals about $5 \%$, predominantly the RCP component is generated, and the degree of polarization in the integral SSP flux is over $35 \%$.

The similarity of the time profiles obtained independently at the SSRT and with the NAOC spectropolarimeters, confirms the correctness of the measurements of the temporal and spectral features of the microwave pulse. A comparison of the spectra and signals at separate frequencies clearly shows that the time profile of the signal obtained in the narrow band is determined primarily by the spectrum dynamics.

\section{Coordinate measurements}

Interferometric observations permit us to determine of the SSP source position and estimate its size. Two-dimensional images prior to the flare showed one microwave source lying between sunspots of opposite polarity. A next image, taken $10 \mathrm{~s}$ after the start of hard X-ray emission in the HXT/L channel, showed two sources with different signs of circular polarization and centered on spots. The signs of polarization of these sources corresponds to the extraordinary mode and are typical of the sunspot emission. A comparison with images in soft X-ray emission suggests that the main structural element of the flare is the loop. It connects regions nearby magnetic spots of opposite polarity.

The SSP position and size can be determined in two coordinates near the minimum of the U-shaped structure, when the receiving frequencies of the NS and EW arrays are close. During the pulse, the position and shape of the source did not change considerably. The SSP source was located near the RCP background source with a shift of 4-5 thousand kilometers toward the LCP-source. The accuracy of relative referencing of the X-ray and microwave images does not contradict the statement that the SSP source is localized near the brightness source of X-ray emission with high plasma density. 


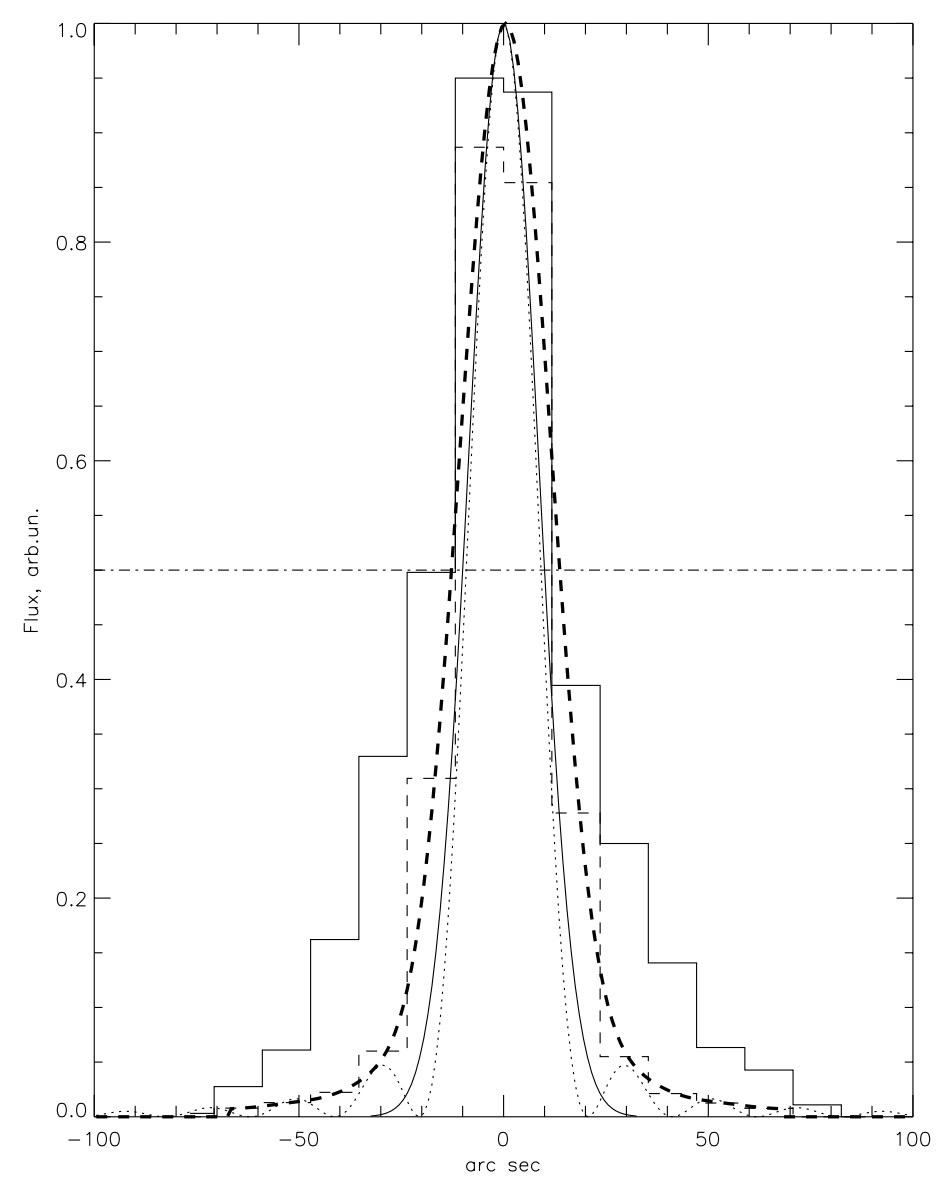

Fig. 6. Analysis of the spatial structure of the SSP source obtained with the NS array. The solid step-like line shows the difference scan (R+L) corresponding to the second panel from top in Fig. 4. Steps correspond to separate frequency channels, the angular distance between them is $10.5^{\prime \prime}$. The dotted line shows the calculated NS beam, and the thin line shows the Gaussian source of a width of 20". Dashed lines show the result of the convolution of the source with the beam: by and without taking into account the cadence of measurements in distance-frequency.

\section{SSP source size}

It is customary to interpret the U-shaped structure of the dynamic spectrum as the reflection of the movement of the electron beam along the flare loop. It is assumed that the emission frequency is controlled by background plasma parameters (the concentration and/or the magnetic field) at the place of emission. At the loop top, the emission frequency must be minimal. An increase in the frequency corresponds to the displacement of the source toward the footpoints of the magnetic loop. In this case the ends of the U-shaped structure at high frequencies, correspond to boundaring of the loop region in which the generation occurs. As is intimated by the curves of Fig. 5 (upper panel), the difference of their positions does not exceed 2" or about one thousand kilometers in EW direction. Consequently, along the EW scanning direction the SSP source is a point-like source with the size on the order of several hundred kilometers.

The assumption about the compactness of sources of subsecond pulses of microwave emission is generally accepted (e.g. review of Benz 1986; Fleishman \& Melnikov 1998). It follows from the conjecture about the coherent nature mechanism of the SSP generation at the eigen-frequencies of background plasma. In this case the size $L=\lambda \Delta f / f$ of the emission region is determined by the band width $\Delta f$ and the uniformity scale $\lambda$ of plasma parameters, and when $\lambda$ is on the order of 10 thousand kilometers it does not exceed several hundred kilometers.

In contradiction with the previous conclusion, the SSP source has an extent no less than $15^{\prime \prime}\left(\approx 10^{9} \mathrm{~km}\right)$ in the direction along the NS scanning direction. This size compares with the length of the flare loop, and at this scale the uniformity of plasma parameters (the density and the magnetic field) that ensures the observed narrow band of emission, is not possible.

The apparent source size reaches a value of about one half of the product of the velocity of light by the growth time of the pulse. First of all, an increase of the size can be caused by the scattering of emission due to plasma density fluctuations in the lower corona. In the microwave range this effect can be significant if the level of plasma density fluctuations far exceeds the equilibrium, thermal level. The effect was estimated by Bastian (1995) and confirmed in the SSRT observations of several tens of subsecond pulses (Altyntsev et al. 1996a). The apparent size of SSP sources increases toward the limb with an increase of the path length of emission through the scattering layer of the lower corona, and can be as large as one min of arc on the limb. However, the contribution of this effect to the apparent source size cannot be decisive because in 17 September event was observed in the central part of the Sun where the scattering is unimportant at angular scales larger than $10^{\prime \prime}$. 


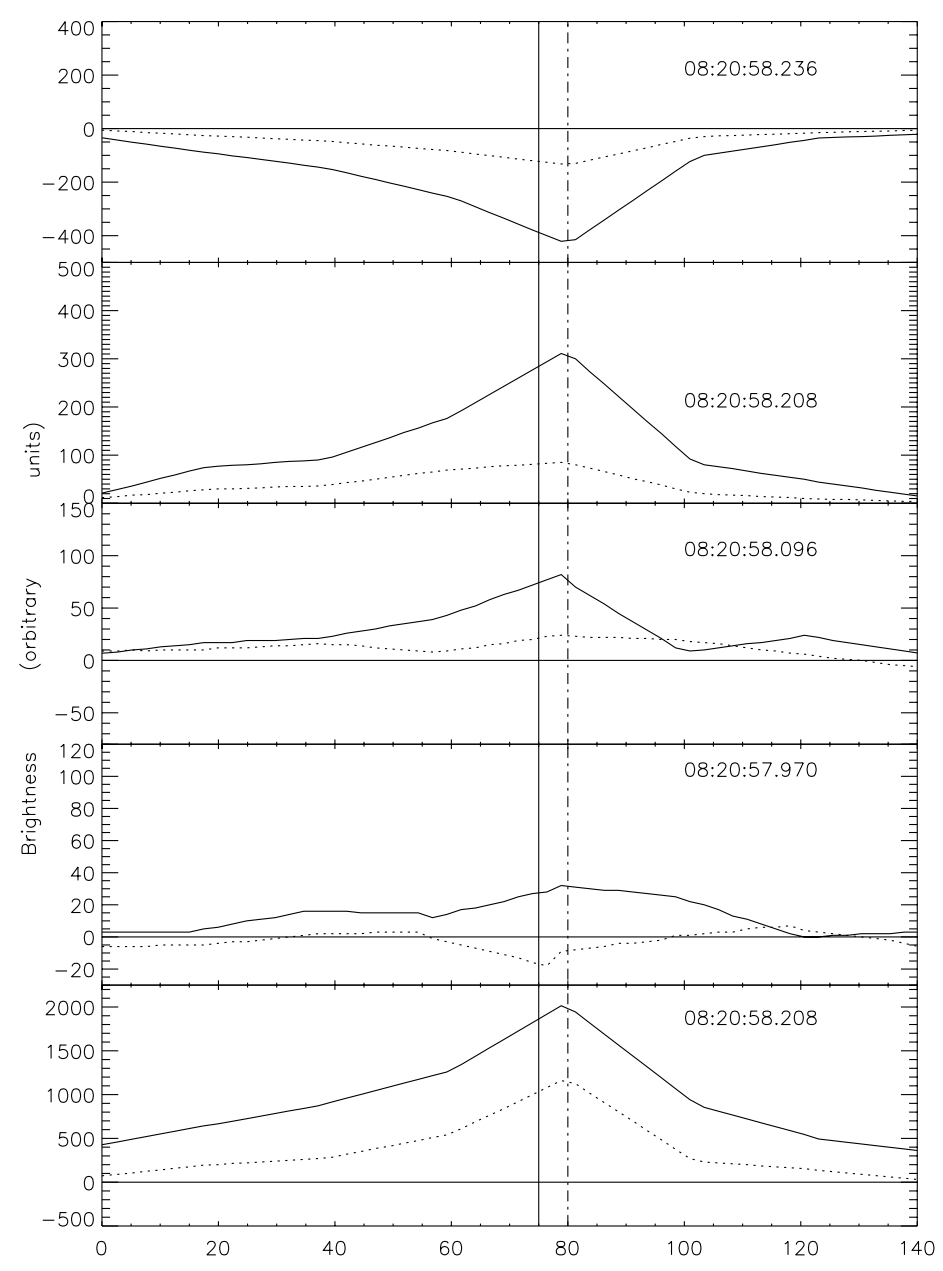

Fig. 7. Same as in Fig. 4 but for the EW array. Solid lines correspond to the brightness distributions as recorded in the 70th order, at frequencies close to the SSP receiving frequency by the NS array.

The large apparent source size on the solar disk can be caused by the reflection and scattering from structural elements with increased plasma density in the environment around the SSP source. The reflected flux must be weaker than the primary one, but the observed values of the brightness temperature can be comparable if the primary emission is an isotropic and is not directed earthward. The anisotropy of SSP emission can be associated both with the emission mechanism and with the conditions of the escape from the SSP source. If the emission frequency is on the order of a local plasma frequency, then a significant anisotropy can be brought about by refraction and absorption of the waves.

As a confirmation of this effect, it is possible to consider the observations of extended footpoints on a one-dimensional brightness distribution of the SSP sources. Their brightness was several times larger than the SSRT beam side lobes. Such pedestals, the relative amplitude and shape of which varied from event to event, were observed by Altyntsev et al. (1996a).

For the reflection from a dense plasma from below, it is possible to study qualitatively the form of the brightness distribution. In this case the source size is determined by its height above the region of reflection and scattering. The result of a convolution of the SSRT beam with the reflection source for a homogeneous underlying surface is shown in Fig. 8. The best agreement between the observed and calculated radio brightness distributions is observed at the source height of about $5.5 \times 10^{8} \mathrm{~cm}$. The distribution falls with the distance from the brightness center slowly, and the agreement of the calculated profile with the measured one is significantly better than for the Gaussian source. The effects of emission reflection from lower layers in the corona were discussed in the analysis of the observations of type III bursts at meter wavelengths (Wild and Smerd 1972).

\section{Emission mechanism}

Assuming the source size of $500 \mathrm{~km}$, the observed flux of $220 \mathrm{sfu}$ corresponds to a brightness temperature of about $10^{10} \mathrm{~K}$. The high value of the brightness temperature and the narrow band of emission suggest a coherent mechanism of emission. Usually, interpretation of the fine structure uses various modifications of the plasma or maser mechanisms of emission (see reviews by Benz 1986; Fleishman \& Melnikov 1998). The energy source is provided by nonthermal electrons, the presence of which follows from hard X-ray observations.

The conditions in which these mechanisms are realized differ greatly. Maser emission is effective when emission is generated at fundamental harmonics of cyclotron frequency, which 


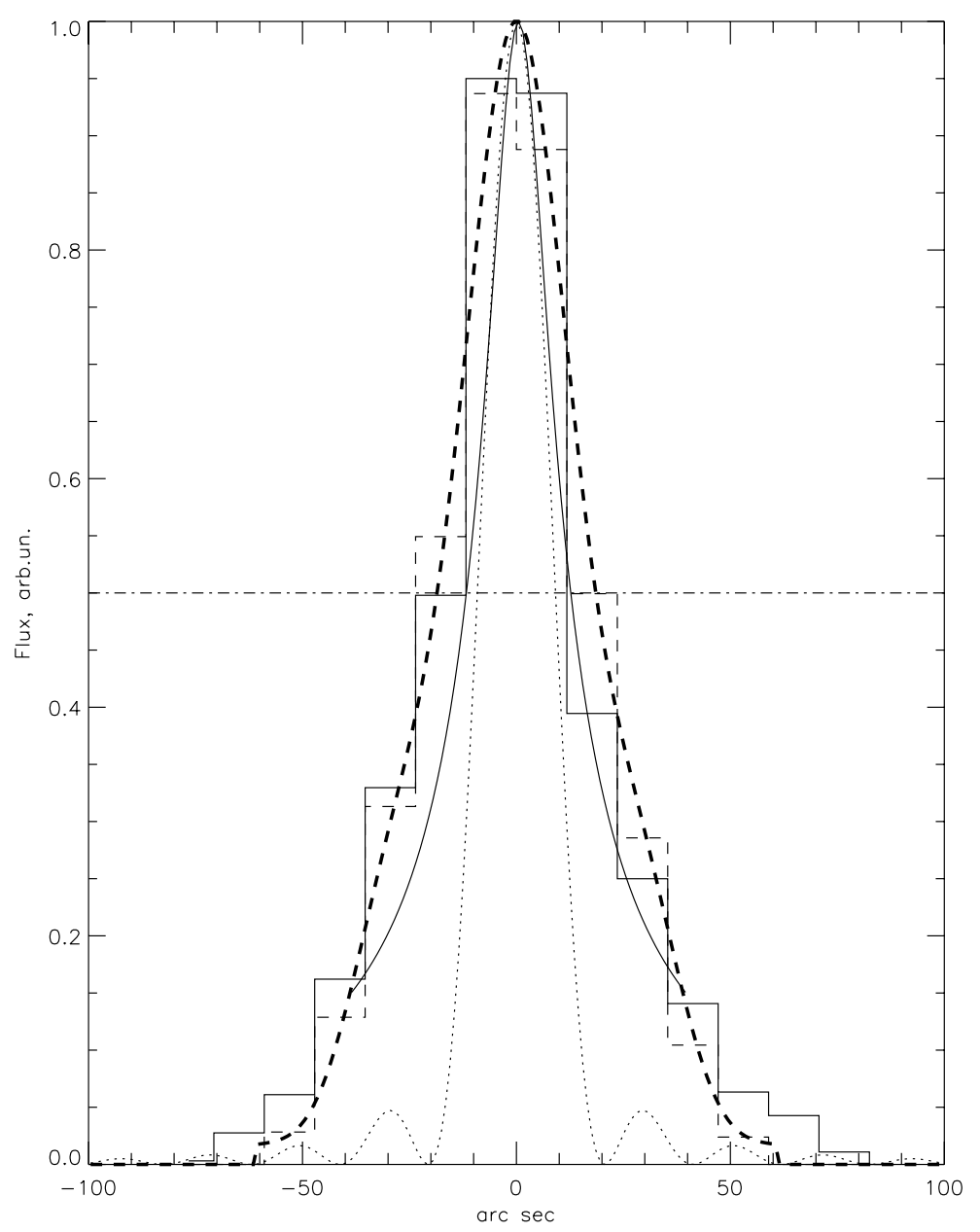

Fig. 8. The same as Fig. 6, but for the reflection source.

requires large values of the magnetic field. In the September 17, 2001 event, the SSP source was located far from the leading sunspot with a large magnetic field. The magnetic field of the $\mathrm{N}$-polarity sunspot, near which the source of the subsecond pulse was located, does not exceed $370 \mathrm{G}$. The SSRT receiving frequency corresponds to the 6th-7th harmonics of cyclotron frequency. This causes us not to consider the maser radiation to be the SSP emission mechanism.

In the plasma mechanism the generation proceeds at frequencies close to the Langmuir frequency $\frac{1}{2 \pi} \sqrt{\frac{4 \pi n e^{2}}{m}}$ or its harmonic, which implies a plasma density in the source $(1-4) \times$ $10^{11} \mathrm{~cm}^{-3}$ at $5.7 \mathrm{GHz}$. In soft X-ray emission at 08:21:06 UT (Yohkoh/SXT) the region in the neighborhood of the southeastern RCP source was the brightest, where the plasma density can exceed the above value.

For determining the emission mechanism, an important information is provided by the polarization of emission. In the analysis of spectrograms it is usually assumed that the magnetic field in the source is determined by the polarity of the leading sunspot. An analysis shows that this conclusion cannot be considered conclusive. As follows from one-dimensional EW scans, the sign of polarization of the background burst source becomes opposite several seconds before the SSP. If this effect is associated with the quasi-perpendicular propagation of electromagnetic waves in the corona above the source, then the sign of polarization of the SSP must also undergo the reversal. Consequently, the conclusion about the generation of ordinary waves is more justified.

\section{Conclusions}

We have described the observations of a subsecond pulse with the relative emission band width of several percent. It has been shown that the SSRT time profiles are controlled by the dynamics of the emission spectrum. The SSP source is located near the following sunspot of the active region and is displaced along the flare loop with respect to the corresponding brightness center of the background burst by $6^{\prime \prime}$. The apparent size of the spike source along the direction of the flare loop is about $15^{\prime \prime}$, and less than $10^{\prime \prime}$ along the other direction.

The narrow band of emission and the high brightness temperature are indicative of a coherent mechanism of spike emission. The frequency of spike generation corresponds to the 6th-7th harmonic of cyclotron emission in the magnitude of the photospheric magnetic field. On the other hand, the brightness center of the flare in soft X-ray emission corresponds to the region of SSP generation, and the estimates of the plasma density in the source do not contradict the assumption about the plasma mechanism of its emission. 
Acknowledgements. We are grateful to Drs G. Chernov and V. Grechnev for useful discussions, and to Mr.Mihalkovskiy for his assistance in preparing the English version of the manuscript. Data used here became available to us thanks to efforts of colleagues operating the Yohkoh and Soho missions. This work was supported by Russian projects of RFBR Nos.00-02-16819, 02-02-06050 and 00-15-96710, Vuz grant (E00-8.0-71), Integratsiy project Nos.I0208 and Astronomiy and Chinese G2000078403 and NSFC.

\section{References}

Altyntsev, A. T., Grechnev, V. V., Konovalov, S. K., et al. 1996a, ApJ, 469,976
Altyntsev, A. T., Dutov, A. A., Grechnev, V. V., et al. 1996b, Sol. Phys., 168, 145

Altyntsev, A. T., Nakajima, H., Takano, T., \& Rudenko, G. V. 2000, Sol. Phys., 195, 401

Benz, A. O. 1986, Sol. Phys., 104, 99

Benz, A. O., Graham, D., Isliker, H., et al. 1996, A\&A, 305, 970

Fu, Q., Qin, Z., Ji, H., \& Pei, L. 1995, Sol. Phys., 160, 97

Gary, D. E., Hurford, G. J., \& Flees, D. 1991, ApJ, 369, 255

Fleishman, G. D., \& Melnikov, V. F. 1998, Physics Uspekhi, 41, 1157

Shibasaki, K., Takano, T., Enome, S., et al. 1994, Space Sci. Rev., 68, 217

Takano, T., \& the Nobeyama Radioheliograph group 1994, Proc. of Eighth International Symposium on Solar-Terrestrial Physics, Sendai, Japan, 44

Wild, J. P., \& Smerd, S. F. 1972, ARA\&A, 10, 159 